\title{
TeV Scale Spontaneous R-Parity Violation ${ }^{1}$
}

\author{
Pavel Fileviez Pérez and Sogee Spinner \\ Department of Physics, University of Wisconsin, Madison, WI 53706, USA
}

\begin{abstract}
Understanding the origin or absence of the $R$-parity violating interactions in the minimal supersymmetric Standard Model is a vital and open issue. Here we show that in the minimal $B-L$ models, $R$-parity and $B-L$ are spontaneously broken at the TeV scale. We also briefly discuss the phenomenological and cosmological aspects of these scenarios.
\end{abstract}

Keywords: Physics beyond the Standard Model, Supersymmery, R-Parity, B-L Symmetry

PACS: $12.60 . \mathrm{Cn}, 12.60 . \mathrm{Jv}$

\section{INTRODUCTION}

Supersymmetry (SUSY) has captured the imagination of many of the practitioners of our field due to the elegant solution to the hierarchy problem and rich phenomenology. An open issue in SUSY is the origin of lepton (L) and baryon (B) number violating interactions:

$$
\hat{L} \hat{H}_{u}, \quad \hat{L} \hat{L} \hat{E}^{C}, \quad \hat{Q} \hat{L} \hat{D}^{C}, \quad \hat{U}^{C} \hat{D}^{C} \hat{D}^{C} .
$$

Since the phenomenological and cosmological aspects of the MSSM hinges on the presence or absence of these interactions, it is crucial to understand the status of the so-called $R$-parity symmetry. $R$-parity is defined as $R=(-1)^{3(B-L)+2 S}=(-1)^{2 S} M$, where $M$ is called matter parity and $B, L$ and $S$ stand for baryon number, lepton number and spin, respectively. Conservation of $R$-parity forbids the dangerous processes due to the operators listed above, e.g. the dimension four contributions for proton decay, and guarantees the stability of the lightest supersymmetric particle (LSP), a good candidate for the cold dark matter of the Universe. Although even if $R$-Parity is broken, the gravitino still makes a good dark matter candidate. Therefore, understanding the origin or absence of $R$-parity conservation is key to appreciating the phenomenological and cosmological implications of the SUSY program. This is the main focus of our letter which discusses our findings published in Refs. [1], [2], [3] and [4].

Examining the definition of $M$-parity leads one to imagine several scenarios related to $B-L:$ i) if $B-L$ is conserved, $M$ is always conserved. ii) If $B-L$ is broken by an even number, $M$ is conserved, and iii) if $B-L$ is broken by an odd number, $M$-Parity is violated. These scenarios can be realized in either global or local $B-L$ symmetries. Global $B-L$ has been studied in the past [5] and necessitates facing the Majoron problem [6], while a local, $R$-parity conserving approach, was investigated in a systematic way in Ref. [7]. Here we focus our attention on the latter case of local $B-L$ symmetries

\footnotetext{
${ }^{1}$ Based on a plenary talk given by P.F.P. at PPC09, OU, May 2009 and talk given by S.S. at SUSY09, Northeastern Univ., Boston, June 2009.
} 
and show that the minimal $B-L$ model always requires the spontaneous breaking of R-Parity.

We have investigated this idea for the origin of the $R$-parity violating interactions in four different scenarios [1, 2, 3, 4] where the electroweak gauge group at the TeV scale is

- a) $S U(2)_{L} \otimes U(1)_{Y} \otimes U(1)_{B-L}$

b) $S U(2)_{L} \otimes U(1)_{Y} \otimes U(1)_{(a Y+b(B-L))}$,

- c) $S U(2)_{R} \otimes S U(2)_{L} \otimes U(1)_{B-L}$,

d) $S U(2)_{L} \otimes U(1)_{I_{3}^{R}} \otimes U(1)_{B-L}$.

In all these theories $B-L$ is part of the local symmetry and the minimal matter content includes only the MSSM states plus three generations of right-handed neutrinos (required by anomaly cancellation). If no new states are added in by hand, the only way to break the gauge symmetry down to the SM gauge symmetry must also break $R$ parity spontaneously. This is achieved through the vacuum expectation value of the righthanded sneutrino. The resulting theory is a simple TeV scale extension of the MSSM, with the following attractive properties:

1. A mechanism for spontaneous $R$-parity violation $(\mathrm{SRpV})$, which generates only bilinear terms.

2. The $R$-parity and $B-L$ violating scales are defined by the SUSY mass scale (TeV scale).

3. Rapid proton decay is avoided since Baryon number is preserved at the renormalizable level.

4. Neutrino masses are generated at tree level through $R$-parity violation and the type I seesaw mechanism.

5. Testability at the LHC through the $Z^{\prime}$ properties and $R$-parity violating decays.

6. The gravitino as a viable dark matter candidate.

In the next section we discuss in detail the mechanism for spontaneous $R$-parity violation in these scenarios.

\section{LOCAL $B-L$ SYMMETRY AND SRPV}

In the minimal $B-L$ extension of the MSSM the gauge group of the electroweak sector is based on $G_{B-L}=S U(2)_{L} \otimes U(1)_{Y} \otimes U(1)_{B-L}$. The particle content and its $G_{B-L}$ charges are given by

$$
\begin{aligned}
& \hat{Q}^{T}=(\hat{U}, \hat{D}) \sim(2,1 / 3,1 / 3), \quad \hat{U}^{C} \sim(1,-4 / 3,-1 / 3), \\
& \hat{D}^{C} \sim(1,2 / 3,-1 / 3), \quad \hat{L}^{T}=(\hat{N}, \hat{E}) \sim(2,-1,-1), \\
& \hat{E}^{C} \sim(1,2,1), \quad \hat{N}^{C} \sim(1,0,1),
\end{aligned}
$$

and as we know the MSSM Higgses have no $B-L$ charge. In this case the most general superpotential is

$$
\mathscr{W}=\mathscr{W}_{\mathrm{MSSM}}+Y_{v} \hat{L} \hat{H}_{u} \hat{N}^{C}
$$


with

$$
\mathscr{W}_{\mathrm{MSSM}}=Y_{u} \hat{Q} \hat{H}_{u} \hat{U}^{C}+Y_{d} \hat{Q} \hat{H}_{d} \hat{D}^{C}+Y_{e} \hat{L} \hat{H}_{d} \hat{E}^{C}+\mu \hat{H}_{u} \hat{H}_{d}
$$

The soft SUSY breaking potential contains the terms

$$
\begin{aligned}
V_{\text {soft }} & \supset M_{\tilde{N}^{C}}^{2}\left|\tilde{N}^{C}\right|^{2}+M_{\tilde{L}}^{2}|\tilde{L}|^{2}+M_{\tilde{E}^{C}}^{2}\left|\tilde{E}^{C}\right|^{2}+m_{H_{u}}^{2}\left|H_{U}\right|^{2}+m_{H_{d}}^{2}\left|H_{D}\right|^{2} \\
& +\left(A_{v} \tilde{L} H_{u} \tilde{N}^{C}+\text { h.c. }\right) .
\end{aligned}
$$

At this point, it may seem like an extra field is needed in order to break the gauge symmetry to the SM. However, the minimal model already contains the necessary ingredient, the right-handed neutrino. Therefore in the minimal $B-L$ model R-parity is spontaneously broken. We proceed by investigating the scalar potential. Once one generation of sneutrinos, $\tilde{v}$ and $\tilde{v}^{C}$ and the Higgses acquire vacuum expectation values (VEVs) $v_{L}, v_{R}$ and $v_{u, d}$ (with $v_{u}^{2}+v_{d}^{2}=v^{2}$ ) respectively, the components of the scalar potential reads as

$$
\begin{aligned}
\left\langle V_{F}\right\rangle & =\frac{1}{4} Y_{v}^{2}\left(v_{R}^{2} v_{u}^{2}+v_{R}^{2} v_{L}^{2}+v_{L}^{2} v_{u}^{2}\right)+\frac{1}{2} \mu^{2} v^{2}-\frac{1}{2} Y_{v} \mu v_{d} v_{L} v_{R}, \\
\left\langle V_{\mathrm{soft}}\right\rangle & =\frac{1}{2}\left(M_{\tilde{L}}^{2} v_{L}^{2}+M_{\tilde{N}^{C}}^{2} v_{R}^{2}\right)+\frac{1}{\sqrt{2}} A_{v} v_{u} v_{L} v_{R}, \\
\left\langle V_{D}\right\rangle & =\frac{1}{32} g_{B L}^{2}\left(v_{R}^{2}-v_{L}^{2}\right)^{2} .
\end{aligned}
$$

where we have ignored the pure MSSM Higgs contributions.

Minimizing in the limit $v_{R} \gg v_{u}, v_{d} \gg v_{L}$

$$
v_{R}=\sqrt{\frac{-8 M_{\tilde{N}^{C}}^{2}}{g_{B L}^{2}}}, \quad v_{L}=\frac{v_{R} B_{v}}{M_{\tilde{L}}^{2}-\frac{1}{8} g_{B L}^{2} v_{R}^{2}},
$$

and $B_{v}=\frac{1}{\sqrt{2}}\left(Y_{v} \mu v_{d}-A_{v} v_{u}\right)$. Notice that the expression for the right-handed sneutrino $\mathrm{VEV}$ is extremely simple in this case and resembles that of the SM. It also indicates that the right-handed sneutrino needs a negative soft mass squared to achieve symmetry breaking. While this is possible through some modifications of the variety of popular SUSY breaking mechanisms available, it is important to note that it is also possible through the inclusion of Fayet-Iliopoulos terms for $G_{B-L}$. Regardless, it is crucial to mention that the $R$-parity breaking scale is defined by the SUSY breaking scale.

$R$-parity Violation: After symmetry breaking, the effective MSSM-like theory will contain $R$-parity violating bilinear terms, all of which will be defined by two VEVs: $v_{L}$ and $v_{R}$, where $v_{R} \gg v_{L}$. For example, the $Y_{v} \hat{L}^{T} i \sigma_{2} \hat{H}_{u} \hat{N}^{C}$ term in the superpotential, leads to $Y_{v} l \tilde{H}_{u} v_{R} / \sqrt{2}$ and $Y_{v} \tilde{H}_{u}^{0} v^{C} v_{L} / \sqrt{2}$. Other generated bilinears, such as mixing between the neutrinos and neutral gauginos, will be proportional to $v_{L}$ and will therefore be quite small, while the mixing between the $B-L$ gaugino and right-handed neutrino will be proportional to $v_{R}$, but will not lead to any dangerous low energy consequences. Notice that only bi-linear $R$-parity violating terms which violate lepton number are 
generated. Therefore, there are no contributions to proton decay at the renormalizable level. See $[3,8]$ for the discussion of the relevant proton decay contributions coming from higher-dimensional operators.

Schematically, the neutrino mass in these types of models have two contributions given by

$$
M_{v}=\frac{1}{2} Y_{v} M_{v^{C}}^{-1} Y_{v} v_{u}^{2}+m^{T} M_{\tilde{\chi}^{0}}^{-1} m,
$$

where the first is a type I seesaw contribution and the second comes from the $R$ parity induced mixings of the neutrinos and neutralinos. Since in both contributions the "seesaw scale" is the TeV, the corresponding Yukawa couplings, $Y_{v}$ must be quite small on the order of $10^{-6}$ or lower. See Refs. [1], [2], [3] and [4] for details.

As was the case for the neutrinos, which mixed with the neutralinos, the charged leptons and sleptons will mix with the charginos and Higgses respectively. However, these mixings are usually proportional to the neutrino mass parameters and are therefore suppressed and do not significantly affect the spectrum. This means that the only deviations from the MSSM spectrum will be the CP-even right-handed sneutrino, corresponding to the $B-L$ breaking VEV (degenerate with the $Z^{\prime}$ gauge boson) and $D$-term contributions to the sfermion masses. Any important deviations from this general discussion will be pointed out below where specific larger gauge groups (containing $B-L$ ) will be considered.

Beyond The Minimal B - L Extension of the MSSM:

- $G_{X}=S U(2)_{L} \otimes U(1)_{Y} \otimes U(1)_{X}$ : The mechanism discussed in the previous section can be applied in a different scenario where $B-L$ is just part of the gauge symmetry. This is the case of a $U(1)_{X}$ extension of the MSSM where $\mathrm{X}$ is a linear combination of weak hypercharge and $B-L, X=a Y+b(B-L)$. The results of our investigation have been published in Ref. [3]. These scenarios are better motivated if one takes into account seriously the idea of Grand Unification because the case $a=1, b=$ $-5 / 4$ corresponds to the embedding in $S O(10)$.

This scenario differs from the previous one discussed before due to the non-zero $X$ charge of the MSSM Higgses, and of course the minimization conditions are different. Doing the same exercise as before we find that

$$
v_{R}=\sqrt{\frac{-8 M_{\tilde{N}^{C}}^{2}-a b g_{X}^{2}\left(v_{u}^{2}-v_{d}^{2}\right)}{g_{X}^{2} b^{2}}},
$$

where the presence of the second term in the numerator could aid $U(1)_{X}$ symmetry breaking, even in the case of a non-negative $M_{\tilde{N}^{C}}^{2}$ although this parameter would need to be very small in that case. In this theory the resulting spectrum is quite different. For example, sfermion masses will have the following contributions from $X D$-terms:

$$
\delta m_{\phi}^{2}=\frac{1}{8} X(\phi)\left[b v_{R}^{2}+a\left(v_{u}^{2}-v_{d}^{2}\right)\right],
$$

where we have ignored contributions from $v_{L}$. The full spectrum of these models have been investigated in Ref. [3] and we invite the reader to study this paper. 
- $G_{L R}=S U(2)_{L} \otimes S U(2)_{R} \otimes U(1)_{B-L}$ : Even more interesting than the Abelian case is the utilization of this mechanism in the context of left-right symmetric models [1], in which the right-handed isospin group and $B-L$ break into the SM hypercharge group. For $R$-parity conservation in this context, an involved Higgs sector is required [9]. Once again though, symmetry breaking and $\mathrm{SRpV}$ can be achieved with just the minimal content. Unfortunately, $G_{L R}$ models need two Higgs bidoublets in order to have a consistent relation between quark masses. This leads to severe constraints coming from flavour violation.

- $G_{I_{3}^{R}}=S U(2)_{L} \otimes U(1)_{I_{3}^{R}} \otimes U(1)_{B-L}$ : An interesting alternative to the previous scenario arises when $G_{L R}$ breaks to $G_{I_{3}^{R}}$ at some high-scale leaving the TeV scale gauge symmetry defined by $G_{I_{3}^{R}}$. In this case it is the combination of the two Abelian groups which breaks into the SM hypercharge. Here, the VEV for the right-handed sneutrinos read as [4]

$$
v_{R}=\sqrt{\frac{-8 M_{\tilde{N}^{C}}^{2}+g_{R}^{2}\left(v_{u}^{2}-v_{d}^{2}\right)}{g_{R}^{2}+g_{B L}^{2}}}
$$

where $g_{R}$ is the $U(1)_{I_{3}^{R}}$ gauge coupling. Here again the $D$-term contributions to the soft masses are different since they do not include the hypercharge contributions that exist in the MSSM. Therefore, in addition to the typical $S U(2)_{L}$ contributions, one also has

$$
\delta m_{\phi}^{2}=\frac{1}{8} B L(\phi) v_{R}^{2}-\frac{1}{8} I_{3}^{R}(\phi)\left(v_{R}^{2}+v_{d}^{2}-v_{u}^{2}\right),
$$

where $B L(\phi)$ is the $B-L$ charge of $\phi$.

Collider Signals: As a consequence of $R$-parity violation, the lightest neutralino will be unstable and will decay via lepton number violating interactions. These interactions will also exist for the charginos and the new gauge boson and can contribute to an interesting scenario which would arise when the sneutrino is the NLSP and the gravitino the LSP. In this case the $Z^{\prime}$ allows for a new production mechanism for sleptons at the LHC:

$$
p p \rightarrow Z^{\prime} \rightarrow \tilde{v} \tilde{v}^{*} \rightarrow e_{i}^{+} e_{j}^{-} e_{k}^{+} e_{l}^{-} \text {. }
$$

Since the sneutrino is the NLSP, it must decay through $R$-parity violating interactions with the following possibilities: $\tilde{v} \rightarrow v v, e_{i}^{+} e_{j}^{-}$. This will lead to channels with four leptons in the final state such as : eеee, $е \mu \mu \mu, e е \mu \mu, e е e \mu, \mu \mu \mu \mu$ and also with several tau's. With such spectacular signals, one could test the existence of $R$-parity violation and lepton number violation. Distinguishing between the different scenarios discussed above can be accomplished by studying the properties of the $Z^{\prime}$ and the different $R$-parity violating decays.

\section{SUMMARY}

The possibility of understanding the origin or absence of the $R$-parity violating interactions in low energy SUSY models has been discussed. Specifically, we have pointed out 
that in minimal models where $B-L$ is part of the local symmetry $R$-parity should be spontaneously broken, along with $B-L$, at the TeV scale. We have discussed this mechanism for spontaneous $R$-parity violation in different extensions of the MSSM, pointing out ways of distinguishing between these at colliders. In these scenarios the gravitino can be the cold dark matter candidate and channels with multi-leptons are crucial for testing this idea at the LHC.

\section{ACKNOWLEDGMENTS}

It is pleasure to thank our collaborators V. Barger and L. Everett for their contributions to the work presented here. The work of P.F.P. was supported in part by the U.S. Department of Energy contract No. DE-FG02-08ER41531 and in part by the Wisconsin Alumni Research Foundation. S.S. is supported in part by the U.S. Department of Energy under grant No. DE-FG02-95ER40896, and by the Wisconsin Alumni Research Foundation. Last, but not least, we would like to thank Espresso Royale for hospitality.

\section{REFERENCES}

1. P. Fileviez Pérez and S. Spinner, Phys. Lett. B 673, 251 (2009).

2. V. Barger, P. Fileviez Pérez and S. Spinner, Phys. Rev. Lett. 102, 181802 (2009).

3. P. Fileviez Pérez and S. Spinner, Phys. Rev. D 80, 015004 (2009).

4. L. L. Everett, P. Fileviez Pérez and S. Spinner, Phys. Rev. D 80 (2009) 055007.

5. A. Masiero and J. W. F. Valle, Phys. Lett. B 251 (1990) 273; J. C. Romao, C. A. Santos and J. W. F. Valle, Phys. Lett. B 288 (1992) 311; M. Shiraishi, I. Umemura and K. Yamamoto, Phys. Lett. B 313 (1993) 89; G. F. Giudice et al, Nucl. Phys. B 396, 243 (1993).

6. G. B. Gelmini and M. Roncadelli, Phys. Lett. B 99 (1981) 411; C. S. Aulakh and R. N. Mohapatra, Phys. Lett. B 119 (1982) 136; Y. Chikashige, R. N. Mohapatra and R. D. Peccei, Phys. Lett. B 98 (1981) 265.

7. See for example: R. N. Mohapatra, Phys. Rev. D 34, 3457 (1986); S. P. Martin, Phys. Rev. D 46 (1992) 2769; Phys. Rev. D 54 (1996) 2340.

8. P. Nath and P. Fileviez Pérez, Phys. Rept. 441 (2007) 191; B. Bajc, P. Fileviez Pérez and G. Senjanovic, Phys. Rev. D 66 (2002) 075005; arXiv:hep-ph/0210374.

9. C. S. Aulakh, A. Melfo and G. Senjanovic, Phys. Rev. D 57 (1998) 4174; R. Kuchimanchi and R. N. Mohapatra, Phys. Rev. D 48 (1993) 4352; K. S. Babu and R. N. Mohapatra, Phys. Lett. B 668 (2008) 404 